\title{
Performance Evaluation of the FLEX Triumph X-PET Scanner Using the National Electrical Manufacturers Association NU-4 Standards
}

\author{
Rameshwar Prasad ${ }^{1}$, Osman Ratib ${ }^{1}$, and Habib Zaidi ${ }^{1,2}$ \\ ${ }^{1}$ Division of Nuclear Medicine, Geneva University Hospital, Geneva, Switzerland; and ${ }^{2}$ Geneva Neuroscience Center, \\ Geneva University, Geneva, Switzerland
}

The purpose of this work was to evaluate the performance characteristics of the preclinical X-PET subsystem of the FLEX Triumph PET/CT scanner based on the NU 4-2008 standards of the National Electrical Manufacturers Association (NEMA). Methods: The performance parameters evaluated include the spatial resolution, scatter fraction, count losses and random coincidences, sensitivity, and image-quality characteristics. The PET detector array consisted of 11,520 individual bismuth germanate crystals arranged in 48 rings and 180 blocks, with an axial field of view (FOV) of $11.6 \mathrm{~cm}$ and a inner ring diameter of $16.5 \mathrm{~cm}$. The spatial resolution was measured with a small ${ }^{22} \mathrm{Na}$ point source (diameter, $0.25 \mathrm{~mm}$ ) at different radial offsets from the center. Sensitivity was calculated using the same source by stepping the source axially through the axial FOV of the scanner. Scatter fraction and counting-rate performances were determined using a mouse- and rat-sized phantom with an ${ }^{18} \mathrm{~F}$ line source insert. The NEMA image-quality phantom and rodent imaging were also performed to access the overall imaging capabilities of the scanner. Results: Tangential spatial resolution in terms of full width at half maximum varied between 2.2 $\mathrm{mm}$ at the center of the FOV and $2.3 \mathrm{~mm}$ at a radial offset of 2.5 $\mathrm{cm}$. The radial spatial resolution varied between 2.0 at the center and $4.4 \mathrm{~mm}$ at a radial offset of $2.3 \mathrm{~cm}$. The peak system absolute sensitivity was $5.9 \%$ at the center of the FOV. The absolute system sensitivity was 0.67 counts/s/Bq, and the relative total system sensitivity was $73.9 \%$. The scatter fraction for the mouse-sized phantom was $7.9 \%$, with a peak true counting rate of 168 kilocounts per second (kcps) at $0.3 \mathrm{MBq} / \mathrm{mL}$ and a peak noise-equivalent counting rate of $106 \mathrm{kcps}$ at $0.17 \mathrm{MBq} /$ $\mathrm{mL}$. The rat-sized phantom had a scatter fraction of $21 \%$, with a peak true counting rate of $93 \mathrm{kcps}$ at $0.034 \mathrm{MBq} / \mathrm{mL}$ and a peak noise-equivalent counting rate of $49 \mathrm{kcps}$ at $0.02 \mathrm{MBq} / \mathrm{mL}$. Recovery coefficients for the image-quality phantom ranged from 0.13 to 0.88 . Conclusion: The performance of the X-PET scanner based on the NEMA NU 4-2008 standards was fully characterized. The overall performance demonstrates that the $\mathrm{X}$-PET system is suitable for preclinical research.

Key Words: PET; preclinical imaging; performance evaluation; NEMA; rodents

J Nucl Med 2010; 51:1608-1615

DOI: 10.2967/jnumed.110.076125

Received Feb. 11, 2010; revision accepted Jul. 8, 2010.

For correspondence or reprints contact: Habib Zaidi, Division of Nuclear Medicine, Geneva University Hospital, CH-1211 Geneva 4, Switzerland.

E-mail: habib.zaidi@hcuge.ch

COPYRIGHT ๑ 2010 by the Society of Nuclear Medicine, Inc.
1 ET has proven a valuable emerging molecular imaging modality to detect and quantify biochemical processes in vivo. However, as one technology leads to its own advancements, PET is now advancing toward application-specific instrumentation, leading to the design of dedicated highresolution and high-sensitivity clinical brain, prostate, and breast scanners and small-animal PET units for biomedical research. During the last 2 decades, biomedical research has made substantial progress in the use of small-animal models for studying human disease ( 1 ). Molecular imaging using dedicated small-animal PET scanners is now well established in the field of drug development and experimental studies using animal models of various diseases (2). After the development of the first dedicated rodent PET scanner at Hammersmith Hospital in collaboration with CTI PET Systems, Inc., various design concepts of preclinical PET scanners are continually being investigated, developed, and, in some cases, made commercially available through spin-off companies from university research (3-7). With the commercial introduction and widespread availability of preclinical PET systems, small-animal imaging is becoming readily accessible and increasingly popular (8).

The FLEX Triumph PET/CT scanner (Gamma Medica) is the first dedicated trimodality preclinical platform (9). This scanner consists of the X-PET subsystem, a commercialized version of the rodent research PET scanner developed by the M.D. Anderson PET instrumentation group (10), and a micro-CT subsystem. The FLEX system uses a compact solid-state-based SPECT detector and micro-CT subsystem and can be configured with either the X-PET (11) or the LabPET (12) as a PET subsystem.

Both X-PET and CT devices are mounted on the same gantry platform, allowing both functional and anatomic volumes to be imaged without any motion, apart from bed translation, of scanning volumes during the 2 procedures. The development of the X-PET subsystem was guided by key factors such as lower cost, higher sensitivity, and high spatial resolution (10). These factors are the driving force for the design and development of PET scanners dedicated for biomedical research. Usually, the acceptance of any new system is mainly determined by its technical specifications. 
Standardization of the assessment of system performance characteristics is thus highly desired $(13,14)$. Recently, the National Electrical Manufacturers Association (NEMA) published its NU 4-2008 standards for performance evaluation of small-animal PET scanners (15). These standards provide consistent and standardized procedures for measuring scanner performance parameters for small-animal PET. They also document operational PET scanner performance as part of the quality-assurance program. However, until now few papers reported on the performance characterization of dedicated preclinical PET scanners using the NU 4-2008 standards (16-20). This work evaluates the overall performance characteristics of the X-PET subsystem of the FLEX Triumph preclinical PET/CT platform using the NEMA NU 4-2008 standards, including spatial resolution, scatter fraction, count losses and random coincidence measurements, sensitivity, and image-quality characteristics. The overall imaging capabilities of the scanner are also demonstrated through experimental phantom and rodent studies.

\section{MATERIALS AND METHODS}

\section{System Description}

The FLEX Triumph PET/CT platform belongs to the latest generation of preclinical PET/CT systems dedicated to rodent imaging. The X-PET subsystem is based on detector blocks arranged in 48 rings with a $10-\mathrm{cm}$ transaxial field of view (FOV) and 11.6-cm axial FOV (AFOV). The PET detector array consists of 11,520 individual bismuth germanate crystals arranged in 180 blocks. The dimensions of each crystal are $2.32 \times 2.32 \times 9.4 \mathrm{~mm}$ in the axial, transaxial, and radial directions, respectively. This optimal design allows for unique properties such as low cost, high sensitivity, and large AFOV using an inexpensive crystal material arranged in a pixilated ring geometry. This scanner incorporates the photomultiplier-quadrant-sharing (PQS) method, which maximizes the number of crystals per photomultiplier that can be decoded, thus improving the spatial resolution. The sensitivity and light output are maximized using 96\% crystal-packing fraction and no light guides. High-yield pileup event recovery electronic processing technology is used to improve counting-rate performance (21). The detector blocks are circularized by slightly grinding each block on the photomultiplier side into a pentagon and having the 2 end rows of crystals in each block tapered $(10,11)$. PET data are acquired using a $250-$ to $750-\mathrm{keV}$ energy window and 12-ns timing window in list-mode format, which can be binned into 3-dimensional (3D) sinograms corresponding to total and true events, respectively. The 3D sinograms can be further rebinned into 2-dimensional (2D) sinograms using either single-slice rebinning (22) or Fourier rebinning (23) approaches. Images can be reconstructed using either $2 \mathrm{D}$ analytic filteredbackprojection (FBP) or iterative methods, such as the orderedsubsets expectation maximization (OSEM) algorithm (24). The PET subsystem specifications are summarized in Table 1.

\section{Spatial Resolution}

The spatial resolution of a PET scanner expresses its ability to distinguish between 2 small point sources. The spatial resolution is usually characterized by the width of the reconstructed pointspread function of compact radioactive point sources. The width of the point-spread function is here defined by its full width at half maximum (FWHM) and full width at tenth maximum (FWTM). In this study, spatial resolution was measured using ${ }^{22} \mathrm{Na}$ point source having a nominal size of $0.25 \mathrm{~mm}$ and a nominal activity of 1.11 $\mathrm{MBq}$, embedded in an acrylic cube (10.0 mm extent on all sides). Measurements were acquired with the source located at the axial center of the FOV and at one fourth of the AFOV from the center at the following radial distances from the center: 5, 10, 15, and 25 $\mathrm{mm}$. At least $10^{5}$ prompt counts were acquired per measurement. The list-mode data acquired at each location were histogrammed into 3D sinograms after random coincidences were corrected. Differences in detection efficiency were compensated using a selfnormalization method (25). Three-dimensional sinograms were rebinned to $2 \mathrm{D}$ sinograms using Fourier rebinning with a maximum ring difference of 47 . Images were reconstructed using $2 \mathrm{D}$ FBP with no smoothing. FWHM and FWTM of the point-source response function in all 3 directions (radial, tangential, and axial) were determined according to NEMA NU 4-2008 standards (15).

\section{Scatter Fraction, Count Losses, and Random \\ Coincidence Measurements}

We measured the relative system sensitivity to scattered radiation and effects of system dead-time and rate of random

\begin{tabular}{|c|c|c|}
\hline Type & Parameter & Specification \\
\hline Detector & $\begin{array}{c}\text { Scintillator } \\
\text { Crystal dimension } \\
\text { No. of crystals }\end{array}$ & $\begin{array}{c}\text { Bismuth germanate } \\
2.32 \times 2.32 \times 9.4 \mathrm{~mm} \\
8 \times 8=64\end{array}$ \\
\hline Scanner & $\begin{array}{l}\text { No. of blocks } \\
\text { No. of detector rings } \\
\text { Crystals per ring } \\
\text { Total no. of crystals } \\
\text { Photomultipliers } \\
\text { Transaxial FOV } \\
\text { Detector ring inner diameter } \\
\text { AFOV } \\
\text { Timing window }\end{array}$ & $\begin{array}{c}180 \\
48 \\
240 \\
11,520 \\
210(19-\mathrm{mm} \text { diameter }) \\
100 \mathrm{~mm} \\
165 \mathrm{~mm} \\
116 \mathrm{~mm} \\
12 \mathrm{~ns}\end{array}$ \\
\hline Data organization & $\begin{array}{l}\text { No. of } 2 \mathrm{D} \text { sinograms } \\
\text { No. of } 3 \mathrm{D} \text { sinograms } \\
\text { Sinogram size } \\
\text { Sampling distance }\end{array}$ & $\begin{array}{c}95 \\
2,304 \\
255 \times 120 \\
0.4 \mathrm{~mm}\end{array}$ \\
\hline
\end{tabular}


coincidence events at various levels of source activity. Scatter fraction and counting-rate performances (true, random, scatter, and total event rates and the derived noise-equivalent counting rate [NECR]) were measured using 2 phantoms simulating the geometries of a mouse and rat. Both phantoms, specifically fabricated in accordance with NEMA NU 4-2008 standards, were made of a solid, right circular cylinder composed of high-density polyethylene (density, $0.96 \pm 0.1 \mathrm{~g} / \mathrm{cm}^{3}$ ). The mouse-sized phantom was $70 \pm 0.5 \mathrm{~mm}$ long and $25 \pm 0.5 \mathrm{~mm}$ in diameter. A cylindric hole (diameter, $3.2 \mathrm{~mm}$ ) was drilled parallel to the central axis, at a radial distance of $10 \mathrm{~mm}$. The rat-sized phantom had a diameter of $50 \pm 0.5 \mathrm{~mm}$ and a length of $150 \pm 0.5 \mathrm{~mm}$, with a cylindric hole (diameter, $3.2 \mathrm{~mm}$ ) drilled parallel to the central axis at a radial distance of $17.5 \mathrm{~mm}$. The initial activity of $42 \mathrm{MBq}$ for the mouse-sized phantom and $48 \mathrm{MBq}$ for the rat-sized phantom was selected on the basis of previous experiments in which the counting rate was plotted against activity. The phantoms were placed in the center of the FOV (CFOV) parallel to the $z$-axis of the scanner. To measure the coincidence rate at different activity levels, sequential images were acquired every $30 \mathrm{~min}$ until the total activity decayed below $0.1 \mathrm{MBq}$. The duration of each acquisition varied from 180 to $600 \mathrm{~s}$ to compensate for the decay of ${ }^{18} \mathrm{~F}$, thus allowing the collection of over 500,000 prompt counts. Data were acquired and processed according to NEMA NU 4-2008 standards. Corrections for intrinsic radioactivity were not necessary because the system used bismuth germanate as scintillation crystal (15).

The total event rate $R_{T O T, i, j}$ for each acquisition $j$ of each slice $i$ is computed as:

$$
R_{T O T, i, j}=\frac{C_{T O T, i, j}}{T_{a c q, j}}
$$

where $T_{a c q, j}$ is the acquisition time.

The true event rate $R_{t, i,}$ for acquisition $j$ of slice $i$ is computed as:

$$
R_{t, i, j}=\frac{\left(C_{T O T, i, j}-C_{r+s, i, j}\right)}{T_{a c q, j}} .
$$

The random event rate $R_{r, i, j}$ for each acquisition $j$ of slice $i$ was computed as:

$$
R_{r, i, j}=R_{T O T, i, j}-\left(\frac{R_{t, i, j}}{1-S F_{i}}\right) .
$$

The scattered event rate $R_{s, i, j}$ for acquisition $j$ of slice $i$ was computed as:

$$
R_{s, i, j}=R_{T O T, i, j}-R_{t, i, j}-R_{r, i, j} .
$$

The scatter fraction for each acquisition $j$ is computed using the following formula:

$$
S F_{j}=\frac{R_{s, j}}{R_{t, j}+R_{s, j}} .
$$

The NECR $R_{N E C, i, j}$ for each acquisition of each slice $i$ is computed as:

$$
R_{N E C, i, j}=\frac{R_{t, i, j}^{2}}{R_{T O T, i, j}} .
$$

The total system counting rates are computed as the sum of the above-mentioned rates over all slices $i$. The scatter fraction, count losses, and random coincidence analysis was implemented using a program developed in-house and implemented in MATLAB 7.4 (The MathWorks).

\section{Sensitivity}

The sensitivity, which is defined as the fraction of coincident annihilation photon pairs emitted from a source that are detected and counted by the scanner, was measured with the same ${ }^{22} \mathrm{Na}$ point source used in the spatial resolution measurements. The source was positioned at the center of the scanner, both axially and transaxially. Counts were acquired at this location and at positions stepped axially at 5-mm increments, covering the AFOV of the whole scanner. The acquisition time was set to $1 \mathrm{~s}$ for each measurement to ensure the collection of at least 100,000 true events with a random-to-true event rate ratio of less than $1 \%$. A background true event rate was determined by acquiring a dataset (1 s) in the absence of a radioactive source in the FOV. All data were analyzed according to NEMA NU 4-2008 standards (15).

The sensitivity (counts/s/Bq) was calculated as follows:

$$
S_{i}=\left(\frac{R_{i}-R_{B, i}}{A_{c a l}}\right)
$$

where $R_{i}$ and $R_{B, i}$ are the source and background counting rates for acquisition $i$, respectively, and $A_{c a l}$ is the source activity.

The relative (percentage) sensitivity for acquisition $i$ is given by:

$$
S_{A, i}=\frac{S_{i}}{0.9060} \times 100
$$

where 0.9060 is the branching ratio of ${ }^{22} \mathrm{Na}$.

The system sensitivity for mouse and rat phantoms was computed as follows:

$$
\begin{gathered}
S M_{\text {tot }}=\sum_{\substack{\text { central } \\
7 \mathrm{~cm}}} S_{i} ; S R_{\text {tot }}=\sum_{\substack{\text { central } \\
15 \mathrm{~cm}}} S_{i} ; S M_{A, \text { tot }}=\sum_{\begin{array}{c}
\text { central } \\
7 \mathrm{~cm}
\end{array}} S_{A, i} ; \\
S R_{A, t o t}=\sum_{\substack{\text { central } \\
15 \mathrm{~cm}}} S_{A, i} .
\end{gathered}
$$

The total system sensitivity was computed as follows:

$$
S_{\text {tot }}=\sum_{\substack{\text { all } \\ i}} S_{i} ; S_{A, t o t}=\sum_{\substack{\text { all } \\ i}} S_{A, i} .
$$

In addition, the axial sensitivity profile was obtained by plotting the sensitivity, $S_{A, i}$, for each slice.

\section{Image-Quality Characteristics}

The purpose of measuring image quality is to produce images simulating those typically obtained in whole-body imaging studies of small rodents with hot lesions and uniform hot regions containing cold areas. To assess overall image quality and accuracy of corrections, the NEMA NU-4 image-quality phantom was filled thoroughly with $5.3 \mathrm{MBq}$ of ${ }^{18} \mathrm{~F}$ and scanned for $20 \mathrm{~min}$. Acquisition started when the activity decreased to 3.7 MBq. This phantom was specifically fabricated in accordance with NEMA NU 4-2008 standards (15) and is made up of polymethylmethacrylate (internal dimensions: length, $50 \mathrm{~mm}$; diameter, $30 \mathrm{~mm}$ ). A CT scan was acquired for attenuation correction using the X-O CT subsystem of the FLEX Triumph PET/CT scanner, with a tube voltage of $75 \mathrm{kVp}$ and a tube current of $300 \mu \mathrm{A}$. The gantry 
rotated in continuous flying mode. A total of 512 projections were acquired in a full $360^{\circ}$ scan, with $2 \times 2$ binning and $1,184 \times 1,120$ projection matrix size. Images were reconstructed using a modified 3D cone-beam Feldkamp algorithm resulting in a $0.17 \times$ $0.17 \times 0.17 \mathrm{~mm}$ voxel size for a $512 \times 512 \times 512$ image volume CT images were further processed and used for attenuation correction of the corresponding PET data of the image-quality phantom (26). The attenuation-corrected PET sinograms were reconstructed using 2D OSEM (4 iterations, 10 subsets) resulting after interpolation in a $256 \times 256 \times 256$ image matrix with an isotropic voxel size of $0.4 \times 0.4 \times 0.4 \mathrm{~mm}$. A 22.5 -mm-diameter (75\% of active diameter) by $10-\mathrm{mm}$-long cylindric volume of interest (VOI) was drawn over the center of the uniform region of the image-quality phantom. The average activity concentration, maximum and minimum values in the VOI, and the percentage SD (\%SD) were calculated. The reconstructed image slices covering the central $10-\mathrm{mm}$ length of the rods were averaged to obtain a single slice of lower statistical noise. Circular regions of interest were drawn around each rod having diameters corresponding to twice the physical diameter of the rods. The mean and SD of the recovery coefficient (RC) for each rod size was analyzed in accordance with NEMA NU 4-2008 standards (15). The accuracy of attenuation correction was tested by drawing VOIs in the waterand air-filled cylindric inserts. The diameter of the VOI was $4 \mathrm{~mm}$ and encompassed the central $7.5 \mathrm{~mm}$ in length in the axial direction. The ratio of the mean in each cold region to the mean of the hot uniform area was reported as spillover ratio (SOR).

The SD of the RCs is calculated as follows:

$\mathrm{SD}_{\mathrm{RC}}=\sqrt{\left(\frac{\mathrm{SD}_{\text {line profile }}}{\text { average }_{\text {line profile }}}\right)^{2}+\left(\frac{\mathrm{SD}_{\text {background }}}{\text { average }_{\text {background }}}\right)^{2}} \cdot$ Eq. 11

\section{RESULTS}

\section{Spatial Resolution}

The results of the radial, tangential, and axial spatial resolution in terms of FWHM and FWTM (mm) are graphically presented in Figure 1 as a function of radial and axial offsets. The measured data at the CFOV of the scanner $(z=0 \mathrm{~mm})$ and at one fourth of the AFOV from the center $(z=29 \mathrm{~mm})$ were analyzed, as suggested in the NEMA NU 4-2008 standards. The volumetric resolution in the CFOV was $12 \mu \mathrm{L}$. The volumetric resolution increased with increasing radial offset. The volumetric resolution at one fourth of the AFOV was $14 \mu \mathrm{L}$. The radial FWHM varied between $2.0 \mathrm{~mm}$ in the CFOV and $2.3 \mathrm{~mm}$ at a radial offset of $25 \mathrm{~mm}$, whereas the axial FWHM ranged between 2.8 and $3.2 \mathrm{~mm}$ for the same radial offset. The tangential resolution was more or less constant over the whole transaxial FOV for all radial offsets. As expected, the depth of interaction effect causes a degradation of the radial resolution as the radial offset is increased.

\section{Scatter Fraction, Count Losses, and Random Coincidence Measurements}

Figure 2 shows the counting-rate performance of the scanner for the mouse- and rat-sized phantoms. The activity injected in each cylindric phantom was divided by its corresponding volume. The volumes of the mouse and rat

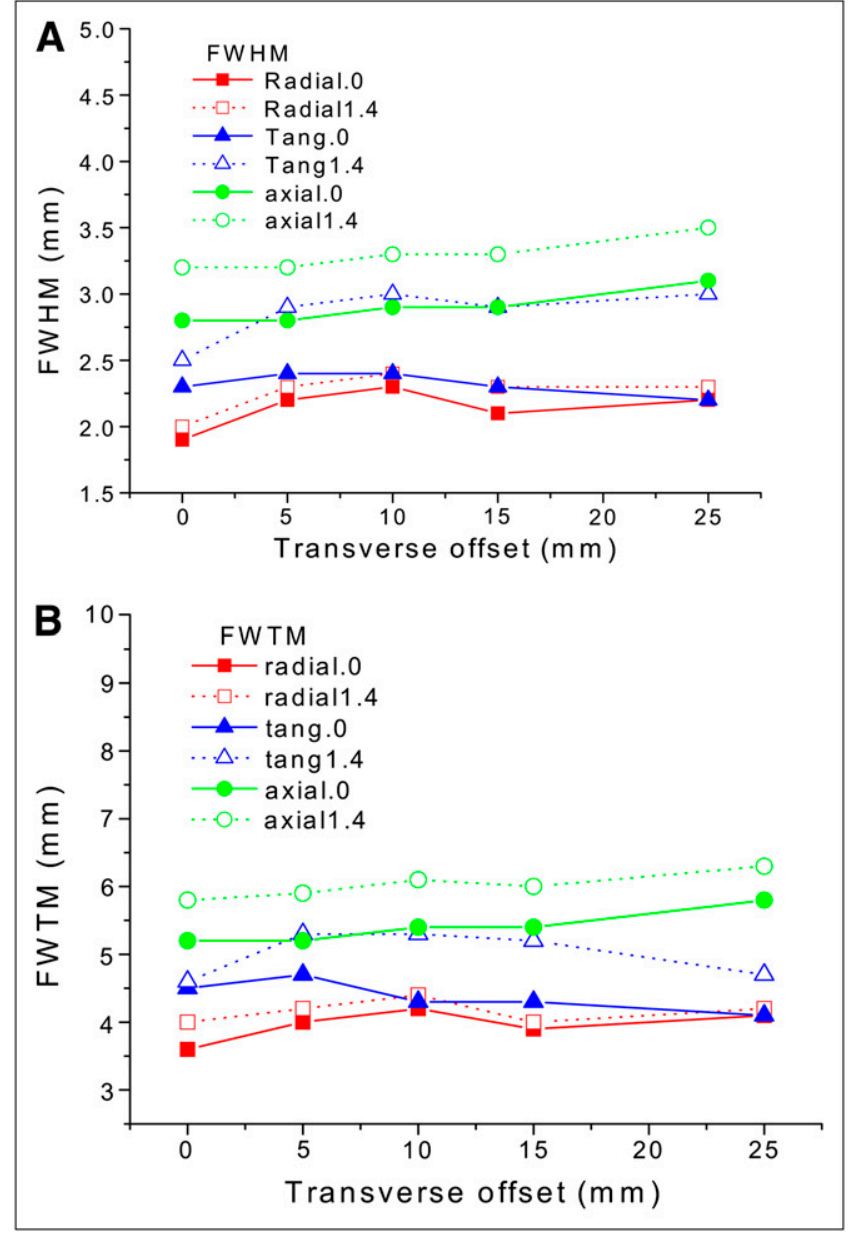

FIGURE 1. Radial, tangential, and axial spatial resolution as function of radial offset reported in terms of FWHM (A) and FWTM (B). Data were measured at 2 axial positions: at center of AFOV and at one fourth from center of AFOV. Tang. = tangential.

phantoms were 34.36 and $294.5 \mathrm{~mL}$, respectively. More specifically, the total, true, random, scatter, and NECRs are plotted as a function of the average effective activity concentration for both mouse- and rat-sized phantoms. The scatter fraction for the mouse-sized phantom was $7.9 \%$, with a peak true counting rate of 168 kilocounts per second (kcps) at $0.3 \mathrm{MBq} / \mathrm{mL}$ and a peak NECR of $106 \mathrm{kcps}$ at $0.17 \mathrm{MBq} / \mathrm{mL}$. The rat phantom had a scatter fraction of $21 \%$, with a peak true counting rate of $93 \mathrm{kcps}$ at 0.034 $\mathrm{MBq} / \mathrm{mL}$ and a peak NECR of $49 \mathrm{kcps}$ at $0.019 \mathrm{MBq} / \mathrm{mL}$.

\section{Sensitivity}

In accordance with NEMA NU 4-2008 standards, the system sensitivity is the sum of the sensitivity calculated for each slice. The total sensitivity for the mouse length (central $7-\mathrm{cm}$ AFOV) was 0.579 counts/s/Bq whereas the total absolute sensitivity was $63.8 \%$. The total sensitivity for the rat phantom (11.4-cm AFOV) was 0.67 counts/s/Bq, whereas the total absolute sensitivity was $73.9 \%$. The system peak absolute sensitivity was $5.9 \%$. The axial 


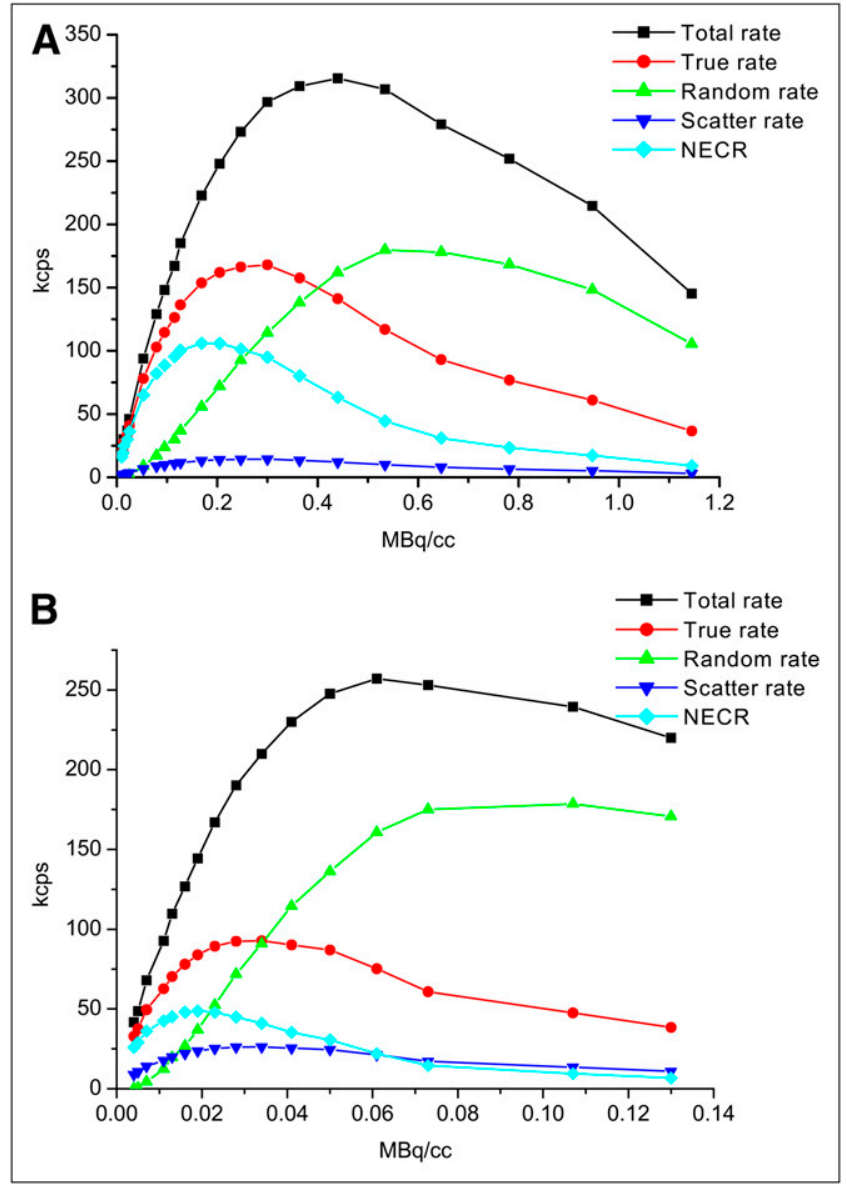

FIGURE 2. Counting-rate performance plots as function of average effective activity concentration of line source for both mouse-sized (A) and rat-sized (B) phantoms.

sensitivity profile obtained by plotting the absolute sensitivity $S_{A}$ for each slice number is shown in Figure 3.

\section{Image-Quality Characteristics}

The sinograms derived from the list-mode file were reconstructed using both 2D FBP and 2D OSEM reconstruction algorithms. The datasets were corrected for attenuation (26) but not for scatter-scatter correction is under development. The mean, maximum, and minimum activity concentration estimates and percentage SD (\% $\mathrm{SD})$ calculated from the drawn VOIs on the uniform section of the image-quality phantom are summarized in Table 2 . The RCs for the different rods along with absolute SD (\% SD) are shown in Figure 4. The SORs obtained for the water- and air-filled inserts of the image-quality phantom are reported in Table 3 along with the \%SD. These values represent the contribution from spillover and scattered events in the reconstructed image of the phantom. Representative images of the NEMA image-quality phantom are shown in Figure 5, illustrating a transaxial view of the 5 rods, a coronal view of the NEMA image-quality phantom, a transaxial view of the uniform region of the phantom, and

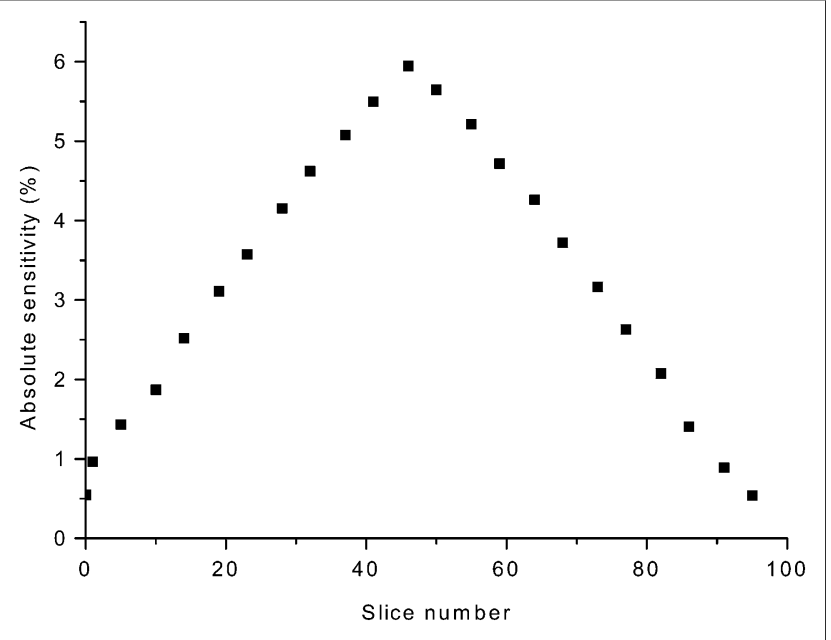

FIGURE 3. Axial absolute sensitivity profile along z-axis of PET scanner using maximum ring difference of 47 .

a profile across the uniform area. The regions of interest defined for quantitative analysis are also shown.

\section{DISCUSSION}

Characterization and performance evaluation are the first and foremost tasks of any PET scanner quality-assurance program. In this work, the overall performance of the FLEX Triumph preclinical PET subsystem was assessed using the newly published NEMA NU 4-2008 standards (15). These standards provide a common platform for measuring the performance of various scanners using specific protocols and guidelines. To the best of our knowledge, there is a lack of published reports on performance evaluation of the FLEX Triumph X-PET system based on NEMA NU 4-2008 standards. However, a few articles documented the performance assessment of other preclinical PET scanners using these standards (16-20).

Spatial resolution results demonstrate that the tangential resolution remains more or less constant over the whole transaxial FOV. Degradation of radial resolution is observed as a function of radial offset. This can be attributed to the depth-of-interaction effect, which leads to larger transaxial parallax errors and hence causes a degradation of the radial resolution as the radial offset increases. The parallax effect-due to crystal penetration in the axial direction

\begin{tabular}{|c|c|c|c|c|}
\hline $\begin{array}{l}\text { Reconstruction } \\
\text { method }\end{array}$ & $\begin{array}{c}\text { Mean } \\
(\mathrm{kBq} / \mathrm{mL})\end{array}$ & $\begin{array}{l}\text { Maximum } \\
(\mathrm{kBq} / \mathrm{mL})\end{array}$ & $\begin{array}{l}\text { Minimum } \\
(\mathrm{kBq} / \mathrm{mL})\end{array}$ & $\begin{array}{l}\% \\
\mathrm{SD}\end{array}$ \\
\hline 2D FBP & 15.70 & 19.51 & 11.16 & 6.37 \\
\hline 2D OSEM & 15.10 & 19.20 & 11.20 & 6.01 \\
\hline
\end{tabular}




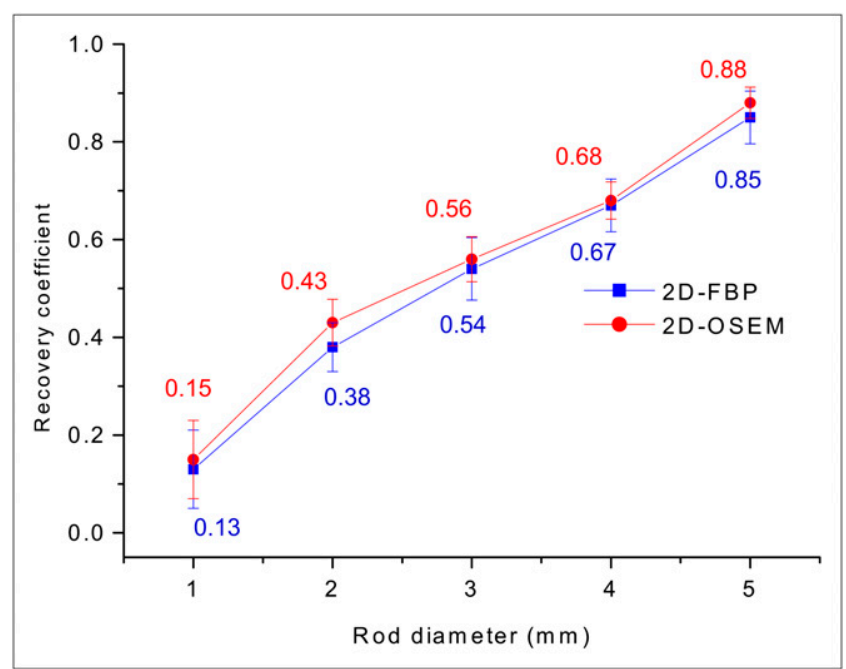

FIGURE 4. RCs and SD for 5 rods of different sizes, ranging between 1 and $5 \mathrm{~mm}$ calculated using both 2D FBP and 2D OSEM reconstruction algorithms.

and the large setting of the maximum ring difference (maximum allowed, $2 \times N_{\text {rings }}-1=47$ ), which is known to degrade spatial resolution (27)—causes deterioration of the axial spatial resolution at large radial offsets when the 3D data are rebinned using a single-slice method (data not shown). The axial spatial resolution can be improved by reducing the maximum ring difference at the cost of reduced sensitivity (28) and also by rebinning the data using Fourier rebinning (Fig. 1). However, the nonuniformity of spatial resolution can be recovered to a great extent using fully 3D statistical iterative reconstruction algorithms through the accurate modeling of PET scanner response $(29,30)$. The obtained results for radial and tangential resolutions are consistent with those reported for the rodent research PET by the M.D. Anderson PET instrumentation development group (31). Moreover, our FWHM values are larger than published results $(17,32)$. Corrections for the size and shape of the source and noncollinearity of positron annihilation were not performed. The spatial resolution results of this system are worse than those reported for other small-animal PET designs having smaller crystal sizes $(17,32)$.

The counting-rate performance was studied in terms of the total, true, random, and scatter event rates, and NECRs, along with scatter fraction. Our results show that the X-PET has excellent counting-rate performance thanks to high-

\begin{tabular}{|c|c|c|c|c|}
\hline \multirow[b]{2}{*}{ Region } & \multicolumn{2}{|c|}{ 2D FBP } & \multicolumn{2}{|c|}{ 2D OSEM } \\
\hline & SOR & $\% \mathrm{SD}$ & SOR & $\% \mathrm{SD}$ \\
\hline Water-filled cylinder & 0.05 & 11.0 & 0.09 & 8.2 \\
\hline Air-filled cylinder & 0.19 & 9.3 & 0.10 & 6.1 \\
\hline
\end{tabular}

yield pileup event recovery electronics. The system achieved a peak true counting rate of $168 \mathrm{kcps}$ at an average activity concentration of $0.3 \mathrm{MBq} / \mathrm{mL}$ and $93 \mathrm{kcps}$ at an average activity concentration of $0.034 \mathrm{MBq} / \mathrm{mL}$ for mouse- and rat-sized phantoms, respectively. The peak NECR was $106 \mathrm{kcps}$ at an average activity concentration of $0.17 \mathrm{MBq} / \mathrm{mL}$ for a mouse-sized phantom and $49 \mathrm{kcps}$ at an average activity concentration of $0.019 \mathrm{MBq} / \mathrm{mL}$ for a rat-sized phantom. The scatter fraction measured at lowactivity concentration for the mouse- and rat-sized phantoms was $7.9 \%$ and $21 \%$, respectively. The NECR for the mouse-sized phantom is higher than the NECR for the ratsized phantom because there is less photon attenuation of the true counting rate in addition to lower scatter and random counting rates. As expected, the NECR for the mouse-sized phantom peaks at a higher activity concentration than the NECR for the rat-sized phantom because the true counting rate decreases more rapidly than the random counting rate as the object size increases (33). Our NECRs for the rat-sized phantom are lower than those reported by Baghaei et al. (31). The reasons for this discrepancy are mainly different PET scanner operational settings and the use of different phantoms (uniform cylinder, compared with a line source in a uniform cylinder in our case); however, more important is the fact that our data were analyzed according to the new NEMA 4-2008 standards, whereas the data of Baghaei et al. (31) were taken directly from the hardware specifications. Saturation of counting rate was observed at an activity concentration of $0.53 \mathrm{MBq} / \mathrm{mL}$ for the mouse-sized phantom and at 0.073 $\mathrm{MBq} / \mathrm{mL}$ for the rat-sized phantom because of the high dead-time of the scanner. However, these activity concentrations are much higher than the activity concentration typically obtained in animal studies. The scatter fraction results are comparable with the results obtained for the Inveon scanner, namely $7.8 \%$ and $17.2 \%$ for a mouseand rat-sized phantoms, respectively (17).

The sensitivity of a PET scanner depends on various factors, such as solid-angle coverage, packing fraction, and scintillator efficiency for 511-keV photons (34). The system peak absolute sensitivity for an energy window of 250-750 $\mathrm{keV}$ and a timing window of $12 \mathrm{~ns}$ is $5.9 \%$, as compared with $9.32 \%$ for the Inveon scanner with an energy window of $250-625 \mathrm{keV}$ and a timing window of $3.432 \mathrm{~ns}$ (17). The axial sensitivity profile shows a linear drop of sensitivity from the center to the edge of the FOV (Fig. 3). The results of the NEMA image-quality tests provide a common and standardized evaluation of the overall image quality achievable by a small-animal PET scanner. The mean VOI values in the uniform region obtained using 2D OSEM reconstructions are slightly lower than those obtained using 2D FBP. Similar trends were reported elsewhere (18). The RCs for 5 different rods ranged from 0.13 to 0.88 , with SD varying between $8 \%$ and $5.4 \%$ (Fig. 4). These RCs are slightly lower than those reported (0.17-0.93) for the Inveon PET scanner (17), in part because our image-quality data were 

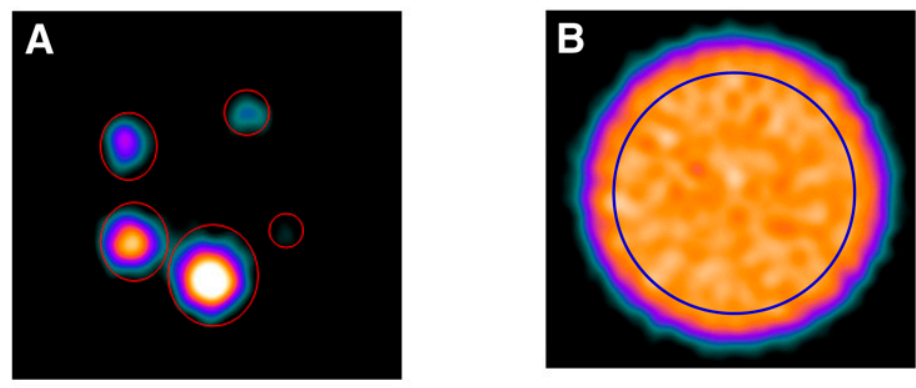

FIGURE 5. Images of NEMA NU-4 image-quality phantom scanned during 20 min with ${ }^{18} \mathrm{~F}(3.7 \mathrm{MBq})$ showing transverse plane corresponding to 5-rod region, with regions of interest defined on each rod $(A)$, transverse plane of uniform region (B), coronal view $(C)$, and line profile across uniform area of phantom (D). Defined regions of interest for quantitative analysis are also shown.
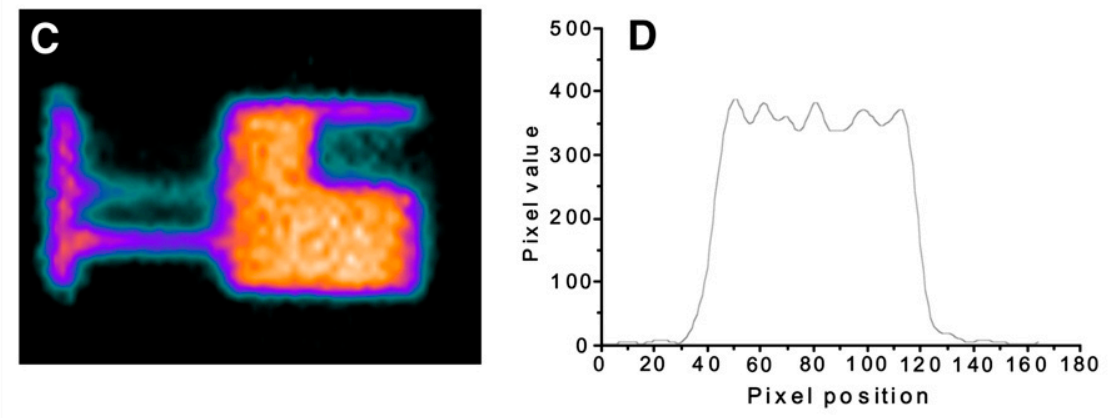

not corrected for scatter. Noncorrection for scatter is being addressed now through the implementation of appropriate CT-based scatter-compensation techniques (35). Moreover, the lower spatial resolution of the system intrinsically results in considerable partial-volume effect. Compared with 2D FBP for all rods, slightly higher RCs and lower $\%$ SDs were obtained for 2D OSEM. SORs of water- and air-filled cylinders for 2D OSEM were 0.09 and 0.10 , with SD of $8.2 \%$ and $6.1 \%$, respectively, which are comparable to corresponding values reported for the MiniPET-II scanner (19). Slightly lower SOR and lower \%SD values were obtained for 2D OSEM than for 2D FBP.

Despite the fact that the FLEX Triumph X-PET scanner is capable of producing high-quality images suitable for molecular imaging research, the performance capabilities of the imaging system that determine the accuracy and precision to which the resulting signal may be measured still need to be improved through reconstruction using accurate statistical modeling and implementation of CTbased PET quantitative correction procedures to compensate for the various image degrading factors including scatter and partial-volume effect.

\section{CONCLUSION}

The performance of the X-PET subsystem of the FLEX Triumph PET/CT scanner has been fully characterized using the newly proposed NEMA NU 4-2008 standards. The results indicate that the scanner has a good spatial resolution across the FOV and a high sensitivity. The counting-rate performance measurements indicate that the scanner is well suited for imaging rodents using low-activity concentrations. The scatter fractions measured for the mouse- and rat-sized phantoms are relatively small. The image-quality phantom results indicate that the image uniformity and RCs are reasonable. However, these values can still be improved by applying appropriate data correction procedures including CT-based scatter and partial volume corrections. Imaging studies of the image-quality phantom and rodents clearly demonstrate good imaging capabilities. The overall performance shows that the FLEX Triumph X-PET system is suitable for preclinical imaging-based research.

\section{ACKNOWLEDGMENTS}

This work was supported by the Swiss National Science Foundation under grant SNSF 3152A0-102143.

\section{REFERENCES}

1. Levin CS, Zaidi H. Current trends in preclinical PET system design. PET Clin. 2007; 2:125-160.

2. Cherry SR, Gambhir S. Use of positron emission tomography in animal research. ILAR J. 2001;42:219-232.

3. Bloomfield PM, Rajeswaran S, Spinks TJ, et al. The design and physical characteristics of a small animal positron emission tomograph. Phys Med Biol. 1995;40:1105-1126.

4. Cherry SR, Shao Y, Silverman RW, Meadors K. MicroPET: a high resolution PET scanner for imaging small animals. IEEE Trans Nucl Sci. 1997;44:11611166.

5. Del Guerra A, Di Domenico G, Scandola M, Zavattini G. YAP-PET: first results of a small animal positron emission tomograph based on YAP:Ce finger crystals. IEEE Trans Nucl Sci. 1998;45:3105-3108.

6. Jeavons AP, Chandler RA, Dettmar CAR. A 3D HIDAC-PET camera with submillimetre resolution for imaging small animals. IEEE Trans Nucl Sci. 1999;46:468-473.

7. Tai C, Chatziioannou A, Siegel S, et al. Performance evaluation of the microPET P4: a PET system dedicated to animal imaging. Phys Med Biol. 2001;46:1845-1862.

8. Stout DB, Zaidi H. Preclinical multimodality imaging in vivo. PET Clin. 2008;3:251-273.

9. Parnham KB, Chowdhury S, Li J, Wagenaar DJ, Patt BE. Second-generation, trimodality pre-clinical imaging system. IEEE Nucl Sci Symp Conf Rec. 2006;3: 1802-1805. 
10. Wong W-H, Li H, Xie S, et al. Design of an inexpensive high-sensitivity rodentresearch PET camera (RRPET). IEEE Nucl Sci Symp Conf Rec. 2003;3:20582062.

11. Xie S, Ramirez R, Liu Y, et al. A pentagon photomultiplier-quadrant-sharing BGO detector for a rodent research PET (RRPET). IEEE Trans Nucl Sci. 2005;52:210-216.

12. Bergeron M, Cadorette J, Beaudoin JF, et al. Performance evaluation of the LabPET APD-based digital PET scanner. IEEE Trans Nucl Sci. 2009; $56: 10-16$.

13. Zaidi H, ed. Quantitative Analysis in Nuclear Medicine Imaging. New York: Springer; 2006.

14. Weber S, Bauer A. Small animal PET: aspects of performance assessment. Eur J Nucl Med Mol Imaging. 2004;31:1545-1555.

15. National Electrical Manufacturers Association. Performance Measurements of Small Animal Positron Emission Tomographs. NEMA standards publication NU 4-2008. Rosslyn, VA: National Electrical Manufacturers Association; 2008.

16. Popota FD, Aguiar P, Fernandez Y, et al. Comparison of NEMA NU 4-2008 vs NEMA NU 2-2001 for the performance evaluation of the microPET R4 system. IEEE Nucl Sci Symp Conf Rec (NSS/MIC). 2009:2706-2709.

17. Bao Q, Newport D, Chen M, Stout DB, Chatziioannou AF. Performance evaluation of the Inveon dedicated PET preclinical tomograph based on the NEMA NU-4 standards. $J$ Nucl Med. 2009;50:401-408.

18. Lage E, Vaquero JJ, Sisniega A, et al. Design and performance evaluation of a coplanar multimodality scanner for rodent imaging. Phys Med Biol. 2009;54: 5427-5441.

19. Kis SA, Lajtos I, Emri M, et al. Performance test of the MiniPET-II small animal scanner according to the NEMA NU-4 standard. IEEE Nucl Sci Symp Conf Rec (NSS/MIC). 2009:3185-3189.

20. Bahri MA, Plenevaux A, Warnock G, Luxen A, Seret A. NEMA NU4-2008 image quality performance report for the microPET Focus 120 and for various transmission and reconstruction methods. J Nucl Med. 2009;50:1730-1738.

21. Wong W-H, Li H, Uribe J, Baghaei H, Wang Y, Yokoyama S. Feasibility of a high-speed gamma-camera design using the high-yield-pileup-event-recovery method. J Nucl Med. 2001;42:624-632.
22. Daube-Witherspoon ME, Muehllehner G. Treatment of axial data in threedimensional PET. J Nucl Med. 1987;28:1717-1724.

23. Defrise M, Kinahan PE, Townsend DW, Michel C, Sibomana M, Newport DF. Exact and approximate rebinning algorithms for 3-D PET data. IEEE Trans Med Imaging. 1997;16:145-158.

24. Hudson HM, Larkin RS. Accelerated image reconstruction using ordered subsets of projection data. IEEE Trans Med Imaging. 1994;13:601-609.

25. Zhang Y, Li H, Baghaei $\mathrm{H}$, et al. A new self-normalization method for PET [abstract]. J Nucl Med. 2008;49:62P.

26. Prasad R, Ay MR, Ratib O, Zaidi H. CT-based attenuation correction on the FLEX Triumph ${ }^{\mathrm{TM}}$ preclinical PET/CT scanner. IEEE Trans Nucl Sci. In press.

27. Visser EP, Disselhorst JA, Brom M, et al. Spatial resolution and sensitivity of the Inveon small-animal PET scanner. J Nucl Med. 2009;50:139-147.

28. Kemp BJ, Hruska CB, McFarland AR, Lenox MW, Lowe VJ. NEMA NU 2-2007 performance measurements of the Siemens Inveon preclinical small animal PET system. Phys Med Biol. 2009;54:2359-2376.

29. Panin VY, Kehren F, Michel C, Casey M. Fully 3-D PET reconstruction with system matrix derived from point source measurements. IEEE Trans Med Imaging. 2006;25:907-921.

30. Tohme MS, Qi J. Iterative image reconstruction for positron emission tomography based on a detector response function estimated from point source measurements. Phys Med Biol. 2009;54:3709-3725.

31. Baghaei H, Zhang Y, Li H, et al. GATE Monte Carlo simulation of a highsensitivity and high-resolution LSO-based small animal PET camera. IEEE Trans Nucl Sci. 2007;54:1568-1573.

32. Constantinescu CC, Mukherjee J. Performance evaluation of an Inveon PET preclinical scanner. Phys Med Biol. 2009;54:2885-2899.

33. Watson CC, Casey ME, Bendriem B, et al. Optimizing injected dose in clinical PET by accurately modeling the counting-rate response functions specific to individual patient scans. J Nucl Med. 2005;46:1825-1834.

34. Kim JS, Lee JS, Im KC, et al. Performance measurement of the microPET Focus 120 scanner. J Nucl Med. 2007;48:1527-1535.

35. Zaidi H, Koral KF. Scatter modelling and compensation in emission tomography. Eur J Nucl Med Mol Imaging. 2004;31:761-782. 